\title{
Adherence to infection control practices in relation to neonatal care in major hospitals in a district of Sri Lanka
}

\author{
Chintha Jayasinghe $^{1^{*}}$ \& Chrishantha Abeysena ${ }^{2}$ \\ ${ }^{1}$ Epidemiology Unit, Ministry of Health, Sri Lanka: ${ }^{2}$ Department of Public Health, Faculty of Medicine, University \\ of Kelaniya, Sri Lanka
}

"Correspondence: chinthaj@yahoo.com

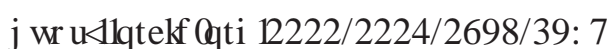

DOI: https://doi.org/10.4038/jccpsl.v25i4.8208

Received on 19 June 2019

Accepted on 5 Oct 2019

\begin{abstract}
Introduction: Adherence to infection control practices are crucial for neonatal care.

Objectives: To describe the adherence to clean birth and postnatal care practices by healthcare workers in selected procedures in major hospitals in a district of Sri Lanka

Methods: This was a qualitative observational study. The study sample comprised healthcare workers (doctors, nurses and midwives) attached to the labour room (LR), postnatal ward (PNW), neonatal intensive care unit (NICU) and operating theatre (OT). A total of 70 healthcare workers from the LR, 90 from the PNW, 50 from the NICU and 60 from the OT were assessed in relation to each procedure they carried out. Four checklists were used to record the observations on infection control standards. The performance of each healthcare worker for each specific procedure was observed only once.

Results: Hand washing practices among the healthcare workers in LR, NICU, PNW and OT varied, with better practices seen in NICU and PNW. Recapping of the needles was done by $18.6 \%, 18.0 \%$ and $31.2 \%$ in LR, NICU and PNW, respectively. Disposal of sharps to the bin was done by almost all healthcare workers in the LR, NICU and PNW, respectively. All the healthcare workers used 70\% alcohol to disinfect the skin, of whom the majority used povidone iodine when drawing blood samples for blood culture in the NICU. Changing or washing gloves before cutting the umbilical cord was observed in the majority of healthcare workers in LR and the minority in OT.
\end{abstract}

Conclusions: Adherence to infection control standards by healthcare workers in observed procedures was not up to the standard.

Key words: birth, infection, hand washing, postnatal, neonates, sepsis 


\section{Introduction}

Infection control is a series of procedures and guidelines that are followed to prevent healthcare associated infections. There are infections that are acquired by patients during their stay in hospital and infections that are acquired by healthcare workers while working in the hospital. These are known as hospitalacquired infections (HAI). Patients who require treatment for HAI must stay longer in hospitals, are treated with expensive antibiotics and have a high mortality. HAI may be transmitted by healthcare workers to patients, from patient to patient and from patient to healthcare workers (1-2). For every 100 hospitalized patients, seven in the developed and 10 in developing countries will acquire at least one HAI (2). In low- and middle-income countries, the frequency of ICU-acquired infections is at least 2-3-fold higher than in high-income countries (2). New-borns are at higher risk of acquiring HAI in developing countries, with infection rates of 3-20 times higher than in highincome countries (2). It is estimated that at any given time, more than 1.4 million people worldwide are suffering from HAI (3). Infection control therefore has been identified as a model for the emerging patient safety movement.

There are various guidelines to achieve maximum control of HAI (4-6). These guidelines should always be followed by all healthcare workers when attending on patients, regardless of their diagnosis or presumed infectious status. These guidelines mainly address subjects such as hand hygiene, use of personal protective equipment, management of sharps, management of blood spills, and handling of blood, body fluids and specimens (6). A systematic review concluded that clean birth and postnatal care practices were effective in reducing neonatal mortality from sepsis (7).

HAI also impose a great burden on hospital resources. Adherence to the guidelines is therefore of paramount importance to prevent infections in the hospital setting. In a setting where resources are poor, infection can exceed $20 \%$ but the World Health Organisation (WHO) declares that available data are scanty, and more research is urgently needed to assess the burden of disease in developing countries (3). Therefore, it is important to find out the current situation on infection control practices among healthcare workers for the improvement of neonatal care. The objective of this study was to describe the adherence to infection control practices in relation to neonatal care by healthcare workers in selected procedures in the labour rooms (LR), neonatal intensive care units (NICU), postnatal wards (PNW) and operating theatres (OT) in secondary and tertiary care hospitals in a district of Sri Lanka.

\section{Methods}

This was a qualitative observational study carried out in all four major hospitals in the district of Gampaha, namely Colombo North Teaching Hospital, District General Hospital Gampaha, District General Hospital Negombo and Base Hospital Wathupitiwala from July 2010 to February 2011. In the four hospitals, there were seven LR, five NICU, nine PNW and six OT conducting caesarean section. The study sample comprised healthcare workers in the relevant units, namely doctors, nursing sisters, nursing officers and midwives.

Ten healthcare workers were selected from each unit of the four hospitals to assess the adherence to infection control related to procedures, thus a total of 70 healthcare workers from LR, 90 from PNW, 50 from NICU and 60 from OT were recruited. The performance of each healthcare worker on each identified procedure was observed only once. Seven types of procedures were observed in the LR; seven in the NICU; two in the PNW; and five in the OT.

Four checklists were developed to record the observations on infection control standards in the four types of units. The checklists were prepared using the following documents; WHO Essential New-born Care Course, Integrated Management of Pregnancy and Childbirth (8), the LR Management Guidelines of the Ministry of Health (9) and Infection Control Manual by the College of Microbiologists (6). Essentially, the checklist assessed the use of personal protective equipment (PPE) by healthcare workers, adherence to infection control standards when performing normal vaginal delivery and caesarean section, hand hygiene and management of sharps, provision of new-born care including cord care and breastfeeding management and practices, management of blood spills, examination of new born babies, blood drawing/IV cannulations for neonates, and management of specimens such as blood 
and urine samples and procedures when performing BCG vaccinations. The checklists were reviewed by a group of experts including specialists in paediatrics, microbiology, public health and gynaecology. The face $\&$ content validity and the appropriateness of the checklists were assessed by the experts. The check lists were further pre-tested in a hospital in Colombo District for feasibility and appropriateness.

It was decided to observe selected procedures from each unit on randomly selected dates. All units were visited during shifts according to a roster prepared in advance. During each shift, only one or two procedures were observed from the same unit. Staff in the institution was unaware of the dates the observations were carried out. Ten such observations were made for each selected procedure from each unit. Procedures were selected to represent all relevant categories of staff. The checklist was filled shortly after the procedure was over.

\section{Data analysis}

Data analysis was done using the Statistical Package for Social Sciences (SPSS) version 16. Each item of the checklists included only two responses (Yes or No) and descriptive analysis was carried out.

\section{Results}

\section{Adherence to infection control standards in the labour room}

As shown in Table 1, all healthcare workers ( $\mathrm{n}=70$; $100.0 \%$ ) wore sterile gloves before attending on deliveries in the LR. Change of shoes before entering the LR was practised by $62.9 \%$. Before attending on deliveries, aprons were worn by $80 \%$ while only $57.1 \%$ washed their hands using either bar soap or liquid soap. Removal of bangles, rings and watches was practised by $52.5 \%(\mathrm{n}=21)$ while $100 \%$ washed over the surface of the hands. Majority ( $\mathrm{n}=39 ; 97.5 \%)$ washed the back of the hands, $77 \%(\mathrm{n}=31)$ washed inter-digital spaces, $75 \%(n=30)$ washed back of the fingers, $62.5 \%(n=25)$ washed their thumbs separately and $70.0 \%(\mathrm{n}=28)$ washed the tip of fingers. The majority $(n=37 ; 95.7 \%)$ of healthcare workers used single use sterile towels to wipe their hands after washing.

The used sharps were disposed into sharp bins by $90 \%$ of healthcare workers, whereas recapping before disposing was done by only $18.6 \%$ of healthcare workers. The majority (92.9\%) of them dried the baby immediately but only $41.4 \%$ of babies were kept over the abdomen for skin to skin contact. All healthcare workers used sterile instruments to cut umbilical cords, whereas $7.1 \%$ umbilical cords were cut without proper clamping of the cords. Umbilical cord stump observation for oozing was done for $75.7 \%$ of deliveries.

Leaving the baby over the mother's chest was done during $36.8 \%$ of the deliveries and covering the baby well in $92.9 \%$ deliveries. Although removing of vernix is not practised now, it was observed in four neonates $(5.7 \%)$. Even though the best practice of breastfeeding is to initiate it during 30 minutes to onehour period, it was observed only in $65.7 \%$ of neonates. More than $80 \%$ of mothers were helped to position the neonates for breastfeeding by the healthcare staff. TCL on blood spilled floor was applied only $11.4 \%$ of the times. Separate mops to wipe out the contaminated places were used $71.4 \%$ of the times.

\section{Adherence to infection control standards in NICUs}

As shown in Table 2, the majority $(96.0 \%)$ of healthcare workers changed their shoes when entering the NICU. No one wore sterile caps while in the NICU. Only $60 \%$ of healthcare workers washed hands before entering the NICU. While all healthcare workers washed their hands with soap after touching the sick neonates, only $80 \%$ of them did so before touching the neonates. A Majority $(81.6 \%, n=34)$ of healthcare workers washed the back of the fingers, whereas only $76.8 \%$ $(n=32)$ workers washed the thumbs separately and the tip of fingers. All of them washed over the surfaces of their hands, $94.7 \%(n=39)$ washed back of the hand, and $87.5 \%(\mathrm{n}=36)$ inter digital spaces. There were $78.1 \%(n=32)$ of healthcare workers who wiped their hands using a single use sterile towel, whereas $21.9 \%$ $(n=9)$ wiped their hands using disposable towels.

All the healthcare workers performed cannulation after disinfection of the skin with $70 \%$ alcohol. Hands were washed by $86.0 \%$ before the insertion of cannula, whereas $50.0 \%$ wore sterile gloves. All the healthcare workers used $70 \%$ alcohol for skin disinfection and sterile needles before drawing blood for blood culture. 
Table 1. Adherence to infection control practices by birth attendants in the labour room $(n=70)$

\begin{tabular}{llllll}
\hline Practices & \multicolumn{3}{c}{ Yes } & \multicolumn{2}{c}{ No } \\
& No. & & $\%$ & No. & $\%$ \\
\hline
\end{tabular}

Practices of personal protective equipment and hand washing

Changed the shoes

Wore masks

$\begin{array}{crrr}44 & 62.9 & 26 & 37.1 \\ 9 & 12.9 & 61 & 87.1 \\ 56 & 80.0 & 14 & 20.9 \\ 9 & 12.9 & 61 & 87.1 \\ 40 & 57.1 & 30 & 42.9 \\ 70 & 100.0 & 0 & 100.0\end{array}$

Wore apron

Wore gown

Hand washed before wearing gloves

Wore sterile gloves

Practice of handling sharps while assisting for deliveries

Recap before disposal of syringes

Disposing the syringes without separating the needle

Sharps disposed into sharp bin

\begin{tabular}{rrrr}
13 & 18.6 & 57 & 81.4 \\
66 & 94.3 & 4 & 5.7 \\
63 & 90.0 & 7 & 10.0 \\
2 & 2.9 & 68 & 97.1 \\
\hline
\end{tabular}

\section{Practices while delivering the baby}

Delivered the neonate over the mother's abdomen

Dried the baby immediately

Wiped eyes and mouth

$\begin{array}{cccr}29 & 41.4 & 41 & 58.6 \\ 65 & 92.9 & 5 & 7.1 \\ 60 & 85.7 & 10 & 14.3 \\ 0 & 0.0 & 70 & 100.0 \\ 61 & 87.1 & 9 & 12.9\end{array}$

Applied antimicrobial to eyes

Discarded wet cloths immediately

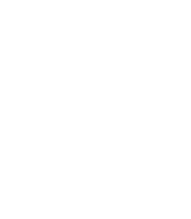

\footnotetext{
Practices while providing umbilical cord care

Changed or washed the gloves before cord was cut

Clamped and cut the cord

Used sterile instruments

Observed for oozing

Application of any substances over the stumps
}

$\begin{array}{rrrr}54 & 77.1 & 16 & 22.9 \\ 65 & 92.9 & 5 & 7.1 \\ 70 & 100.0 & 0 & 0.0 \\ 53 & 75.7 & 17 & 24.3 \\ 0 & 0.0 & 70 & 100.0\end{array}$

cut

\section{Practice just after birth of neonate}

Left the baby on mother's chest for skin to skin contact after delivery

Covered the baby well

$\begin{array}{llcr}27 & 36.8 & 42 & 61.4 \\ 65 & 92.9 & 5 & 7.1 \\ 44 & 62.9 & 26 & 37.1 \\ 66 & 94.3 & 4 & 5.7\end{array}$

Covered the head of the baby

\section{Practices on breastfeeding}

Initiation of breast feeding within $1 / 2$ hour to 1 hour

Help in positioning of the neonates

Help in attachment of the baby

Any other substances given other than breast milk

$\begin{array}{rrrr}46 & 65.7 & 24 & 34.3 \\ 57 & 81.4 & 13 & 18.6 \\ 54 & 77.1 & 16 & 22.9 \\ 0 & 0.0 & 70 & 100.0\end{array}$

\section{Practices taken to prevent cross infection}

Apply TCL and keep it for 30 minutes over the blood spilled floor

Use separate mop to wipe out the contaminated places

Change the mackintosh for the delivery of a new mother

Keep the contaminated materials in the separate bins

$\begin{array}{cccr}8 & 11.4 & 62 & 88.6 \\ 50 & 71.4 & 20 & 28.6 \\ 69 & 98.6 & 1 & 1.4 \\ 69 & 98.6 & 1 & 1.4\end{array}$


Table 2. Adherence to infection control practices by staff in the neonatal intensive care units (NICU) $(n=50)$

Practices
Practice when entering the NICU
Change the shoes
Wear sterile masks
Wear sterile gown
Wear sterile caps
Wash hands before entering the NICU

\begin{tabular}{|c|c|c|c|}
\hline \multicolumn{2}{|c|}{ Yes } & \multicolumn{2}{|c|}{ No } \\
\hline No. & $\%$ & No. & \\
\hline
\end{tabular}

\title{
Practice when handling the neonates
}

Washed hands before handling the neonates

\begin{tabular}{crrr}
48 & 96.0 & 2 & 4.0 \\
2 & 4.0 & 48 & 96.0 \\
17 & 34.0 & 33 & 66.0 \\
0 & 0.0 & 50 & 100.0 \\
30 & 60.0 & 20 & 40.0 \\
\hline
\end{tabular}

Already nails cut short

$\begin{array}{rrrr}41 & 80.0 & 10 & 20.0 \\ 46 & 92.0 & 4 & 8.0 \\ 50 & 100.0 & 0 & 0.0\end{array}$

Washed hands after touching the neonates

Practices when performing venipuncture on the neonates

Wash hands before inserting the cannulas

Wear a pair of sterile gloves before commencing the puncture

Use of $70 \%$ alcohol to clean the skin

$\begin{array}{rrrr}43 & 86.0 & 7 & 14.0 \\ 25 & 50.0 & 25 & 50.0 \\ 50 & 100.0 & 0 & 0.0 \\ 26 & 52.0 & 24 & 48.0\end{array}$

Documentation of date on the BHT/dressing

$\begin{array}{rrrr}50 & 100.0 & 0 & 0.0 \\ 36 & 72.0 & 14 & 28.0 \\ 34 & 68.0 & 16 & 32.0 \\ 48 & 96.0 & 2 & 4.0 \\ 49 & 98.0 & 1 & 2.0 \\ 50 & 100.0 & 0 & 0.0\end{array}$

\section{Practices when drawing blood samples for blood cultures}

Use of $70 \%$ alcohol to clean the skin

Allow to dry

Use of Betadine for cleaning

Wash hands before drawing blood samples

Use of sterile gloves

Use of sterile syringes and needles

(1)

Practice when handling the sharps and disposal of sharps

Recap before disposing syringes

Disposed syringes without separating the needle

Sharps disposed to sharp bins

Change of sharps from person to person

Sharp bins not filled more than $3 / 4$

\begin{tabular}{rrrr}
9 & 18.0 & 41 & 82.0 \\
50 & 100.0 & 0 & 0.0 \\
50 & 100.0 & 0 & 0.0 \\
0 & 0.0 & 50 & 100.0 \\
41 & 82.0 & 9 & 18.0 \\
\hline
\end{tabular}

\section{Practices during hand washing}

Advice mother to hand wash before handling the baby

Supervision of hand washing of mothers

Advice mothers to hand wash after handling the babies

$\begin{array}{crcr}27 & 54.0 & 23 & 46.0 \\ 5 & 10.0 & 45 & 90.0 \\ 12 & 24.0 & 38 & 76.0 \\ 50 & 100.0 & 0 & 0.0\end{array}$

Supply of clean sterile cup for breast milk extraction

\section{Practices when handling the blood and specimens with body fluids}

Place specimens in a leak proof container

$\begin{array}{rrrr}50 & 100.0 & 0 & 0.0 \\ 41 & 82.0 & 9 & 18.0 \\ 50 & 100.0 & 0 & 0.0 \\ 21 & 42.0 & 29 & 58.0\end{array}$


There were $96.0 \%$ of healthcare workers who washed their hands prior to wearing gloves. All the health care workers disposed the sharps into sharp bins and all of them did not separate the needle from syringes. No one $(0 \%)$ changed the sharps from person to person, whereas $18.0 \%$ did the recapping of needles. Twentyseven mothers $(54.0 \%)$ were advised by nurses to wash their hands before handling their sick neonates in the NICU. Only $10.0 \%$ of mothers were supervised by the healthcare workers for hand washing. All placed the specimens in the leak proof container whereas $18.0 \%$ of specimens were contaminated outside the container. Only $42 \%$ of specimens were transported securely to prevent spillage.

\section{Adherence to infection control standards in the postnatal wards}

As shown in Table 3, there were $82.5 \%$ of healthcare workers who washed their hands before attending the neonates in the postnatal wards. Among those who washed hands before attending to the neonates in the postnatal wards, $23.8 \%(n=17)$ removed bangles, $60.8 \%(\mathrm{n}=40)$ used soap, $39.2 \%$ $(n=26)$ used hand rubs, $49 \%(n=35)$ closed the tap by using their elbow or through someone else and $57.5 \%$ $(n=38)$ wiped their hands after washing. Out of the 66 observations of hand washing and hand rubs, 71.4\% $(n=47)$ washed over the surface of the hand, $92.7 \%$ $(n=61)$ washed the back of the hands, $72.9 \% \quad(n=48)$ washed back of the fingers, $71.4 \%(n=47)$ washed inter digital spaces, $63.8 \%(n=42)$ washed thumbs separately, and $60.8 \%,(n=40)$ washed the tips of the fingers.

\section{Adherence to infection control practices related to neonatal care in operating theatre}

As shown in Table 4 , the majority $(83.3 \%)$ of healthcare workers used nail brushes for the first time for the scrub. All healthcare workers used antiseptic solutions for hand washing. Of them, $76.7 \%$ removed or did not wear jewellery such as rings.

Table 3. Adherence to infection control practices by healthcare workers in the postnatal wards $(n=80)$

\begin{tabular}{|c|c|c|c|c|}
\hline \multirow[t]{2}{*}{ Practices } & \multicolumn{2}{|c|}{ Yes } & \multicolumn{2}{|c|}{ No } \\
\hline & No. & $\%$ & No. & $\%$ \\
\hline \multicolumn{5}{|l|}{ Practice when entering the postnatal wards } \\
\hline Hand wash before attending to the neonates & 66 & 82.5 & 14 & 17.7 \\
\hline \multicolumn{5}{|l|}{ Practices when performing BCG vaccinations } \\
\hline Hand washing before vaccination & 62 & 77.4 & 18 & 22.6 \\
\hline Use of disposable needles & 80 & 100.0 & 0 & 0.0 \\
\hline Discarding of needles without recapping & 25 & 68.8 & 55 & 31.2 \\
\hline Dispose without separating needles & 80 & 100.0 & 0 & 0.0 \\
\hline Dispose into sharp bins & 77 & 96.2 & 3 & 3.8 \\
\hline Change sharps from person to person & 0 & 0.0 & 0 & 0.0 \\
\hline Washed hand after vaccination & 80 & 100.0 & 0 & 0.0 \\
\hline
\end{tabular}


Table 4. Adherence to infection control practices by healthcare workers in the operation theatres $(n=60)$

\begin{tabular}{|c|c|c|c|c|}
\hline \multirow[t]{2}{*}{ Practices } & \multicolumn{2}{|c|}{ Yes } & \multicolumn{2}{|c|}{ No } \\
\hline & No. & $\%$ & No. & $\%$ \\
\hline \multicolumn{5}{|l|}{ Practice before attending the caesarean section } \\
\hline Used nail brushes for the firsthand scrub & 50 & 83.3 & 10 & 16.7 \\
\hline Applied antiseptic solutions & 60 & 100.0 & 0 & 0.0 \\
\hline Removed or did not wear jewelry & 46 & 76.7 & 14 & 23.3 \\
\hline Handheld high while washing & 56 & 93.3 & 4 & 6.7 \\
\hline Completely changed into the theatre clothes & 52 & 86.7 & 8 & 13.3 \\
\hline Caps properly covered the hair & 55 & 91.7 & 5 & 8.3 \\
\hline Masks completely covered the nose and mouth & 44 & 73.3 & 16 & 26.7 \\
\hline Wore double gloves & 41 & 68.3 & 19 & 31.7 \\
\hline Masks changed after each operation & 10 & 16.7 & 50 & 83.3 \\
\hline \multicolumn{5}{|l|}{ Practices when entering the Operation Theatre } \\
\hline Completely changed into the theatre clothes & 0 & 0.0 & 60 & 100.0 \\
\hline Changed the shoes when entering the theatre & 60 & 100.0 & 0 & 0.0 \\
\hline Caps properly covered the hair & 45 & 75.0 & 15 & 25.0 \\
\hline Masks completely covered the nose and mouth & 41 & 80.3 & 19 & 31.7 \\
\hline Washed hands before wearing the gloves & 20 & 33.3 & 40 & 66.7 \\
\hline Wore sterile gloves & 60 & 100.0 & 0 & 0.0 \\
\hline
\end{tabular}

Practices by theatre nursing officers when assisting

the caesarean sections

Wiped eyes of the neonates immediately

$\begin{array}{llll}46 & 76.7 & 14 & 23.3\end{array}$

Wiped mouth of the neonates immediately

$\begin{array}{llll}50 & 83.3 & 10 & 16.7\end{array}$

Dried the baby immediately

Practices when performing the neonatal care in the operation theatre

Changed or washed the gloves before cutting the umbilical cord

$\begin{array}{rrcr}19 & 31.7 & 41 & 68.3 \\ 59 & 98.3 & 1 & 1.7 \\ 43 & 71.7 & 17 & 28.3 \\ 60 & 100.0 & 0 & 0.0 \\ 0 & 0.0 & 60 & 100.0 \\ 52 & 86.7 & 8 & 13.3 \\ 0 & 0.0 & 60 & 100.0\end{array}$

Clamped and cut the cord

Observed for oozing

Used sterile instrument

Application of substances over the stump

Discarded wet clothes immediately

Application of antimicrobials to the eyes

\section{Neonates who received the following care practices}

Place the baby on the mothers' abdomen or in

her arms for skin to skin contact

$\begin{array}{rrcr}6 & 10.6 & 54 & 90.0 \\ 60 & 100.0 & 0 & 0.0 \\ 38 & 63.3 & 22 & 36.7 \\ 26 & 43.3 & 34 & 56.7\end{array}$

Started breastfeeding in the theatre itself

43.3 
Fifty-two (86.7\%) healthcare workers had completely changed into theatre clothes when carrying out caesarean section, while $55(91.7 \%)$ and $73.3 \%$ of workers properly wore caps and masks, respectively. All the birth attendants were public health midwives from the relevant wards. None of them changed their uniform when entering the surgical theatre but all changed shoes. Only $33.3 \%$ of birth attendants washed their hands before wearing sterile gloves for handling the neonates.

A majority (76.7\%) of nursing officers wiped the eyes of neonates immediately and the mouth of $83.3 \%$ of neonates. Baby was dried immediately by $81.7 \%$. Only $31.7 \%$ birth attendants changed or washed their hands before cutting the umbilical cord. An observation for oozing was done by $71.7 \%$ of birth attendants. Discarding of wet clothes was done immediately by $86.7 \%$ of birth attendants. All the neonates were started on breastfeeding in the theatre itself, whereas only $10.6 \%$ of the neonates were kept in contact with the mothers' skin inside the theatre. Thirty-eight mothers $(63.3 \%)$ had brought caps and socks for their neonates but only $43.3 \%$ of them had washed them before dressing the neonates.

\section{Discussion}

In the present study, hand washing practices observed among healthcare workers varied across the study units. It was higher in NICU and PNW. It is a well-known fact that HAI spread due to cross infection from poor hand hygiene of healthcare workers, and therefore a simple hand washing procedure is of great consequence in preventing them. The low rates of hand washing reported by midwives might be due to lack of facilities for hand washing in the theatres other than scrubbing area. One Sri Lankan study (10) found that hand washing practices before assisting in the delivery was done by $65 \%$ of the birth attendants. Another study (11) revealed that it was $85.3 \%$. In previous studies, the quality of hand washing had not been evaluated but in the present study, it is found to be poor. The proportion of workers who adhere to the steps of hand washing correctly varied in different settings. When considering the step of washing thumbs separately, the practices in PNW was low. Almost all had used either soap or disinfectant for this purpose, whereas few of them from LR and PNW did not wipe their hands after washing. According to another Sri Lankan study (12), only $10 \%$ of the healthcare workers had overall good practice of hand washing in the ICU set up of a teaching hospital. Doebbling et al. (13) found that the hand washing rate by ICU medical staff was $12.4 \%$ before contact with the patient and $10.65 \%$ after contact. Marito and colleague (14) in Italy also revealed that even after intervention, compliance to hand washing in the NICU among doctors was 50.5\% and $40.7 \%$ among nurses. Another study from Brazil (15) reported that hand hygiene adherence in terms of technique and frequency according to the WHO recommendations was deficient in NICU.

When entering an LR, majority of them wore aprons and minority wore masks. In the NICU, less than $50 \%$ of them wore sterile gowns and few wore masks. In Georgia, the gown and glove compliance in the surgical ICU among doctors was $40 \%$, whereas among nurses it was $68 \%$. In the medical ICU, it was $84 \%$ among nurses and $70 \%$ among doctors (16).

When considering the management of sharps and disposal of sharps, recapping of needles was done by minority of workers in all the units. Disposal of sharps to the sharp bin was done by almost all in the LR, NICU and PNW. One Sri Lankan study (10) also found that only $21 \%$ of healthcare workers practised recapping of needles.

Drawing of blood for cultures is important when managing sepsis babies. When considering the steps in drawing blood, all of the healthcare workers used $70 \%$ alcohol to disinfect the skin, majority of them allowed to dry and used povidone iodine in the NICU.

Skin to skin contact of mother and baby and early initiation of breastfeeding are two important practices to prevent infections in new-borns. In the present study, it was revealed that the majority of neonates were started on breastfeeding within the first hour of delivery, vast majority of healthcare workers helped to position the baby. A study from Sri Lanka (11) found that $98.9 \%$ commenced breastfeeding within the LR while $64.6 \%$ of healthcare workers helped the mothers to breastfeed.

Direct observations using checklists were common practice in similar kind of studies $(10-11,17)$. The presence of an observer while carrying on the procedures may have influenced the practice of the healthcare provider towards favourable direction. To 
minimize this issue, at the beginning of the study, only the nursing officer in-charge was explained regarding the observation of the procedures, but the healthcare providers were unaware of the particular day the observations were carried out. One of the limitations of this study was the inability to obtain in-depth information underling their behaviour. Focus group discussions would be more appropriate for this, in addition to observing the behaviour.

\section{Conclusions \& Recommendations}

The adherence to infection control standards by healthcare workers in observed procedures were not up to the expected standards. During the basic training period, it is necessary to stress on infection control standards for all categories of health staff. At the same time, it is compulsory to introduce regular in-service programmes and conduct routine reviews and evaluations for all types of staff categories regarding infection control.

\section{Public Health Implications}

The adherence to different process of infection control standards by healthcare workers varied. It is compulsory to introduce regular in-service programmes and conduct routine reviews and evaluations for all types of staff categories regarding infection control.

\section{Author Declarations}

Competing interests: The authors declare that they have no conflicts of interests in this study.

Ethics approval and consent to participate: The Ethics Review Committee of the Faculty of Medicine, University of Kelaniya granted ethical clearance. Administrative clearance for the data collection was obtained from the Regional Director of Health Services of Gampaha District and directors of the relevant hospitals prior to data collection. Prior approval was obtained from the consultants in charge of the gynaecology and paediatric wards and the NICU.

Acknowledgements: We are grateful to the Postgraduate Institute of Medicine, University of Colombo, the data collectors and the participants for the study.
Author contributions: CJ participated in the design of the study, coordinated data collection, performed the statistical analysis and helped to draft the manuscript. CA participated in the design of the study, performed the statistical analysis, interpreted the data, drafted the manuscript and approved the final manuscript.

\section{References}

1. Sri Lanka College of Microbiologists. Hospital Infection Control Manual. Colombo: Sri Lanka College of Microbiologists, 2005.

2. WHO. Health Care Associated Infections FACT SHEET.

Available from: https://www.who.int/gpsc/country_ work/gpsc_ccisc_fact_sheet_en.pdf.

3. WHO. Improved hand hygiene to prevent health careassociated infections. Patient Safety Solutions 2009; 9(10).

Available from: https://www.who.int/patientsafety/ solutions/patientsafety/PS-Solution9.pdf.

4. WHO. Guideline on Hand Hygiene in Health Care (Advanced Draft). Global Patient Safety Challenge 2005-2006: Clean Care is Safer Care. Geneva: World Health Organization, 2006. Available from: https://www.who.int/patientsafety/information centre/Last_April_versionHH_Guidelines\% 5B3\%5D.pdf.

5. WHO. Handbook of Integrated Management of Childhood Illnesses. Geneva: World Health Organization, 2005.

Available from: https://apps.who.int/iris/handle/ $10665 / 42939$.

6. Sri Lanka College of Microbiologists. National Guidelines for Infection Control. Colombo: Sri Lanka College of Microbiologists, 2008.

7. Blencowe H, Cousens S, Mullany LC, Lee ACC, Kerber K, Wall S, Darmstadt GL, Lawn JE. Clean birth and postnatal care practices to reduce neonatal deaths from sepsis and tetanus: a systematic review and Delphi estimation of mortality effect. BMC Public Health 2011; 11(Suppl 3): S11.

8. WHO. Integrated management of pregnancy and childbirth. pregnancy, childbirth, postpartum and newborn care: a guide for essential practice. Geneva: World Health Organization, 2003. Available from: https://www.afro.who.int/sites/ default/files/2017-06/mps\%20pcpnc.pdf

9. Family Health Bureau. Guideline for Labour Room Management. Colombo: Ministry of Health, 2007. 
10. Senarath LTUD. Essential newborn care in a district of Sri Lanka and the effectiveness of intervention to improve services. MD Thesis (Community Medicine). Colombo: Post Graduate Institute of Medicine, 2004.

11. Goonewardena CSE. Selected aspect of quality of intrapartum and postpartum care at primary care level institution in the Kalutara district and intervention to improve care. MD Thesis (Community Medicine). Colombo: Post Graduate Institute of Medicine, 2001.

12. Kudavidanage BP, Gunasekara TDCP, Hapuarrachi S. Knowledge, attitudes and practices of hand hygiene among ICU staff in Anuradhapura Teaching Hospital. Anuradapura Medical Journal 2001; 1: 29-40.

13. Doebbling BN, Stanley GI, Sheetz CT. Comparative efficacy of alternative hand washing agents in reducing nosocomial infections in intensive care units. New England Journal of Medicine 1992; 327: 88-93.
14. Marito PA, Ban KM, Bartolone A, Fowler EK, Saint S, Mannelli F. Assessing the sustainability of hand hygiene adherence prior to the patient contact in the emergency department: a one year post intervention evaluation. American Journal of Infection Control 2011;39: 14-18.

15. Silva DS, Dourado AMG, Cerqueira CRE, Romero FH, Amaral NA, Pearce PF, et al. Hand hygiene adherence according to World Health Organization Recommendations in a Neonatal Intensive Care Unit. Revista Brasileira de Saúde Materno Infantil 2017; 17(3): 551-559.

16. Gilbert K, Safford C, Crosby K, Flemiyoe GR. Does hand hygiene compliance among health care workers change when patients are in contact precaution room in ICU. American Journal of Infection Control. 2010; 38: 535-539.

17. Vidal SA, Ronfani L, Silverine SM, Mello MJ, Santoes ER, Buzzetti R, Cattaneo A. Comparison of two training strategeies on essential newborn care in Brazil, Bulletin of the World Health Organization 2001; 79(11): 1024-1031. 